\title{
Targeting Efficacy of Simvastatin for Hormone-Dependent Carcinomas through Solid Lipid Nanoparticles
}

Dasam $\mathrm{U}^{1}$, Natarajan $\mathrm{J}^{1 *}$, Karri VVSR${ }^{1}$, Wadhwani $\mathrm{AD}^{2}$, Antony $\mathrm{J}^{3}$ and Jeyaprakash $\mathrm{MR}^{4}$

${ }^{1}$ Department of Pharmaceutics, JSS College of Pharmacy, JSS University, Ootacamund, Mysuru, India

${ }^{2}$ Department of Pharmaceutical Biotechnology, JSS College of Pharmacy, JSS University, Ootacamund, Mysuru, India

${ }^{3}$ Department of Pharmacology, JSS College of Pharmacy, JSS University, Ootacamund, Mysuru, India

${ }^{4}$ Department of Pharmaceutical Analysis, JSS College of Pharmacy, JSS University, Ootacamund, Mysuru, India

\begin{abstract}
Folate conjugated Simvastatin loaded Solid lipid nanoparticles (SIM-SLNs) were constructed after the preformulation studies such as Differential calorie metry (DSC), Fourier transform infrared spectroscopy (FTIR) and partition coefficient studies. SIM-SLNs were evaluated in terms of particles size, zeta potential, drug encapsulation, and drug release. The cytotoxicity of the SLNs was evaluated on MCF-7 Human Breast Carcinoma cells (MCF-7 cells) by MTT assay. In vivo pharmacokinetic parameters and Bio-distribution were observed in Wistar rats. The particle size of SIM-SLNs (F6) was $177.4 \mathrm{~nm}$ with a zeta potential of $-5.16 \mathrm{mV}$. The entrapment efficiency and drug loading was found to be higher in SIM-SLNs. SLN formulation released the drug in sustained manner over a period of 24 hours. Various kinetic models were fitted and it was observed that Higuchi's model was the best fit model. SIM-SLNs showed highest cytotoxicity in tumor cells in vitro. In vivo studies showed that there is significant increase in the pharmacokinetic parameters and Bio-distribution of SIM-SLNs when compared to the pure drug suspension. Higher relative bioavailability would be due to avoidance of first-pass hepatic metabolism by intestinal lymphatic transport, which circumvents the liver. According to these results, the novel SIM-SLNs provided sustained release of the drug, and these systems are the preferred drug carriers for lipophilic drugs to overcome the oral bioavailability problem of drug and to increase targeting efficacy to the specific organs as breast, prostate and ovary.
\end{abstract}

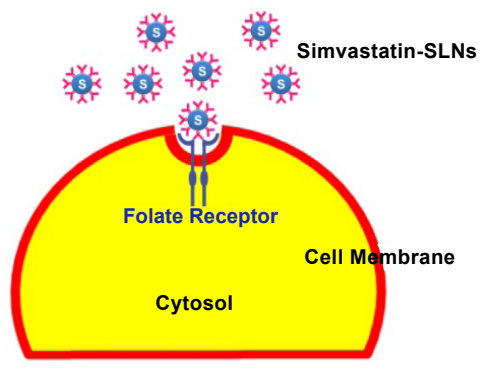

Keywords: Anti-cancer therapy; Solid lipid nanoparticles; Folate conjugated; Targeting effect

\section{Introduction}

Hormone-dependent carcinomas are classically those of reproductive tract- Prostate and Testis cancer in men and Breast, Ovarian and Endometrial cancer in women [1]. Worldwide, breast cancer is the leading type of cancer in women, accounting for $25 \%$ of all cases. In 2012 it resulted in 1.68 million cases and 522,000 deaths. It is more common in developed countries and is more than 100 times more common in women than in men [2]. Breast cancer is a cancer that develops from breast tissue. Prostate cancer, also known as carcinoma of the prostate, is the development of cancer in the prostate, a gland in the male reproductive system.Most prostate cancers are slow growing; however, some grow relatively quickly. The cancer cells may spread from the prostate to other parts of the body, particularly the bones and lymph nodes [3]. Simvastatin, an anti-hyperlipidemic drug shows the anti-cancer activity on hormone dependent carcinomas. The fact that mevalonate plays a key role in cell proliferation and that many malignant cells present an increased HMG-CoA reductase activity, suggests that a selective inhibition of this enzyme could lead to a new chemotherapy for cancer disease. Results obtained in vitro have shown that statins can inhibit tumor cell growth. The obtained reduction of sterols synthesis by statins, suggests that inhibition of tumor cell growth can be related to the reduction of nonsteroidal isoprenoid compounds $[4,5]$. The main challenge in cancer chemotherapy is toxic side-effects induced by chemotherapeutic drugs. Single dose or short-time application (1-2 weeks) will probably causes serious health problems, but the use of biodegradable nano-sized particles for longterm or life-time therapy may produce better therapeutic-effects with less side effects [6,7]. Increasing the encapsulation efficiency of poorly water-soluble molecules will lead to the development of improved SLN formulations and drug-loaded SLN formulations to increase the efficacy and reduce the side-effects of chemotherapeutic drugs for

*Corresponding author: Jawahar Natarajan, Department of Pharmaceutics, JSS College of Pharmacy, JSS University, Ootacamund, Mysuru, India, Tel: 91 9486946314; E-mail: jawahar.n@jsscpooty.org

Received November 24, 2016; Accepted December 22, 2016; Published December 27, 2016

Citation: Dasam U, Natarajan J, Karri VVSR, Wadhwani AD, Antony J, et al. (2016) Targeting Efficacy of Simvastatin for Hormone-Dependent Carcinomas through Solid Lipid Nanoparticles. J Nanomed Nanotechnol 7: 412. doi: 10.4172/21577439.1000412

Copyright: $\odot 2016$ Dasam U, et al. This is an open-access article distributed under the terms of the Creative Commons Attribution License, which permits unrestricted use, distribution, and reproduction in any medium, provided the original author and source are credited. 
Citation: Dasam U, Natarajan J, Karri VVSR, Wadhwani AD, Antony J, et al. (2016) Targeting Efficacy of Simvastatin for Hormone-Dependent Carcinomas through Solid Lipid Nanoparticles. J Nanomed Nanotechnol 7: 412. doi: 10.4172/2157-7439.1000412

Page 2 of 7

anticancer treatment [8]. The purpose of the study is to construct Solid lipid Nanoparticles (SLNs) as nanomedicine for targeting hormonedependent carcinomas by sustained release of the drug and improved oral bioavalabity due to avoidance of first-pass hepatic metabolism through intestinal lymphatic transport, which circumvents the liver.

\section{Materials and Methods}

\section{Materials}

Simvastatin was purchased from IPCA, Mumbai, India. Stearic acid and Tripalmitin were purchased from S.D Fine Chemicals, Mumbai, India. Glyceryl monostearate was purchased from Kemphasol, Mumbai, India. Tween 80 was purchased from Sigma Chemicals, Mumabai, India. Folic acid was purchased from Alpha Chemika, Mumbai, India. Human MCF-7 Breast carcinoma cells (NCCS, Pune, India) were cultured in Dulbecco's Modified Eagle Medium (DMEM) with $10 \%$ fetal bovine serum (FBS) at $37^{\circ} \mathrm{C}$ and $5 \% \mathrm{CO}_{2}$.

\section{Preformulation studies}

Differential scanning calorimetry (DSC): Differential scanning calorimetric analysis was used to characterize the thermal behavior of the drug, lipids and their physical mixtures. Sample was crimped in standard aluminium pans and heated from 20 to $400^{\circ} \mathrm{C}$ at a heating rate of $10^{\circ} \mathrm{C} / \mathrm{min}$ under constant purging of dry nitrogen at $30 \mathrm{ml} /$ min. An empty pan, sealed in the same way as the sample, was used as a reference. DSC thermograms were obtained using an automatic thermal analyzer system. Temperature calibration was performed using Indium calibration reference standard.

Fourier transform infrared spectroscopy (FTIR): FTIR spectroscopy can be used to investigate and predict any physiochemical interaction between different components in a formulation and therefore it can be applied to the selection of suitable chemical compatible excipients while selecting the ingredients, we would chose, those which are stable, compatible, cosmetically and therapeutically acceptable.

Infrared spectra matching approach was used for detection of any possible chemical interaction between the drug, lipid and surfactants. A physical mixture of drug, lipid and surfactants was prepared and mixed with suitable quantity of potassium bromide. This mixture was compressed to form a transparent pellet using a hydraulic press at 15 tons pressure. It was scanned from 4000 to $400 \mathrm{~cm}^{-1}$ in a FTIR spectrophotometer (FTIR 8400 S, Shimadzu). The IR spectrum of the physical mixture was compared with those of pure drug, lipid and surfactants and peak matching was done to detect any appearance or disappearance of peaks.

Partition coefficient studies: Partitioning behavior of Simvastatin(SM) was determined in different lipids viz. stearic acid,glyceryl monostearate and tripalmitin. $10 \mathrm{mg}$ of Simvastatin was dispersed in a mixture of melted lipid $(1 \mathrm{~g})$ and $1 \mathrm{~mL}$ of hot phosphate buffer $\mathrm{pH} 6.8(\mathrm{~PB})$ and shaken for $30 \mathrm{~min}$ in an water bath shaker maintained at $10^{\circ} \mathrm{C}$ above the melting point of lipid. The aqueous phase of the above mixture was separated from the lipid by centrifugation at $10000 \mathrm{rpm}$ for $20 \mathrm{~min}$. The clear supernatant obtained was suitably diluted with phosphate buffer $\mathrm{pH} 6.8$ (PB) and Simvastatin content was determined in UV-Visible spectrophotometer at $239 \mathrm{~nm}$ against solvent blank. The partition coefficient was calculated as:

\section{$\mathrm{PC}=(\mathrm{CSMI}-\mathrm{CSMA}) / \mathrm{CSMA}$}

where, $\mathrm{C}_{\mathrm{SMI}}=$ the initial amount of SM added $(5 \mathrm{mg}$ and $10 \mathrm{mg}$ )
$\mathrm{C}_{\mathrm{SMA}}=$ the concentration of SM in $\mathrm{pH}$ 6.8 PB.

\section{Preparation of solid lipid nanoparticles (SLNs)}

Solid lipid nanoparticles were prepared by solvent evaporation technique. First, o/w microemulsions were prepared. The organic phase was stearic acid or Glyceryl monostearate or tripalmitin. Tween 80 (hydrophilic surfactant) solution was used as continuous phase. The chosen lipid and drug and $10 \mathrm{~mL}$ of ethanol were heated at $60^{\circ} \mathrm{C} .50$ $\mathrm{mL}$ of aqueous surfactant solution containing Tween 80 heated at same temperature and the organic phase was added to the aqueous phase with mechanical stirring for 15 minutes. A clear microemulsion was obtained under stirring at a temperature close to the melting point of the lipid used. Folic acid solution was used as a ligand and added to microemulsion. Solid lipid nanoparticles were obtained by dispersing the warm o/w microemulsion dropwise into ice cold water in a beaker under continuous stirring. SLN dispersion was further stirred after complete addition of microemulsion for 4 hours at 2000 rpm. After completion of stirring, the SLN dispersion was subjected to ultrasonication for 15 minutes. Similarly drug loaded solid lipid nanoparticles were prepared by Solvent evaporation method using stearic acid (SA) and glyceryl monostearate (GMS) and Tripalmitin (TP) as lipids, Simvastatin as drug, Tween 80 was used as hydrophilic surfactant, Folic acid was used as ligand $[9,10]$.

\section{Evaluation of solid lipid nanoparticles}

Particle size and zeta potential: Particle size and zeta potential of the solid lipid nanoparticles were measured by photon correlation spectroscopy using a Malvern Zetasizer Nano ZS90 (Malvern Instruments, Worcestershire, UK), which works on the Mie theory. All size and zeta potential measurements were carried out at $25^{\circ} \mathrm{C}$ using disposable polystyrene cells and disposable plain folded capillary zeta cells, respectively, after appropriate dilution with original dispersion preparation medium.

Polydispersity index: Polydispersity was determined according to the equation,

Polydispersity=D (0.9) $-\mathrm{D}(0.1) / \mathrm{D}(0.5)$

Where,

D (0.9) corresponds to particle size immediately above $90 \%$ of the sample.

D (0.5) corresponds to particle size immediately above $50 \%$ of the sample.

D (0.1) corresponds to particle size immediately above $10 \%$ of the sample [10].

Entrapment efficiency and drug loading: Entrapment efficiency and drug loading of Solid lipid nanoparticles (SLNs) was determined by weighing empty riavials and add $3 \mathrm{~mL}$ of the formulation into riavials. Reweigh it again. Centrifuge the sample for 10 mins with 7000 rpm. After centrifugation take $1 \mathrm{~mL}$ of each supernatant layer of the sample and then make upto $10 \mathrm{~mL}$ with $\mathrm{pH} 6.8$ buffer and do serial dilution and the supernatant liquid absorbance value was determined by UV visible spectroscopy at $239 \mathrm{~nm}$ using $\mathrm{pH} 6.8 \mathrm{~PB}$ as blank.

Entrapment efficiency $(\%)=\frac{\text { Analyzed weight of drug in SLNs }}{\text { Theoretical weight of drug loaded in SLNs }} \times 100$

Drug loading $(\%)=\frac{\text { Analyzed weight of drug in SLNs }}{\text { Analyzed weight of SLNs }} \times 100$ 
External Morphological Study: External morphology of nanoparticles was determined using Scanning Electron Microscopy (SEM). Samples were diluted with ultrapurified water to obtain a suitable concentration. Then the samples were spread on a sample holder and dried using vacuum. They were subsequently coated with gold (JFC 1200 fine coater, Japan) and examined by a Scanning Electron Microscopy (SEM).

\section{In vitro release studies}

The release of Simvastatin from the SLNs was studied under sink conditions. Glyceryl monostearate (GMS-SLN) and Tripalmitin (TPSLN) which showed higher drug content and entrapment efficiency (F3 and F6) were evaluated for in vitro release. $10 \mathrm{~mL}$ of formulation(F3 and F6) were kept in dialysis bags (MWCO 12000, HiMedia). The dialysis bags were placed in $100 \mathrm{~mL}$ of dissolution medium ( $\mathrm{pH}$ 6.8 PB) were kept in orbitory shaker for $24 \mathrm{hrs}$ at $37^{\circ} \mathrm{C}$. Aliquots of the dissolution medium were withdrawn at each time interval and the same volume of fresh dissolution medium was added to maintain a constant volume. Samples withdrawn from $\mathrm{pH} 6.8$ phosphate buffer were analyzed for Simvastatin content spectrophotometrically at $239 \mathrm{~nm}$ against solvent blank.

\section{Haemocompatibility studies}

Blood samples of healthy human volunteers were obtained from blood bank of government hospital, Ooty in evacuated siliconized glass tube containing sodium citrate. Red blood cells were separated by centrifugation at $1500 \mathrm{rpm}$ for $10 \mathrm{~min}$ and then washed 3 times with saline. Stock solution of erythrocytes in Saline water was prepared such that the cell count was $1 \times 108$ cells $/ \mathrm{ml}$. Equal volumes of RBC suspension and nanoparticles dispersion were suspended in a microcentrifuge tube such that the final concentrations of nanoparticle dispersion and nanoparticles were $150-1000 \mu \mathrm{g} / \mathrm{ml}$ and incubated seperately at $37^{\circ} \mathrm{C}$ for $1 \mathrm{~h} .1 \%$ Triton $\mathrm{X}$ and Saline water were used as positive and negative controls respectively. After $1 \mathrm{~h}$ the tubes were centrifuged at $1500 \mathrm{rpm}$ for $10 \mathrm{~min}$ and the hemoglobin released in the supernatant was detected by UV absorbance at $239 \mathrm{~nm}$. All measurements were performed in triplicate $(n=3)$ and the SD was calculated. The percent haemolysis was calculated by the formula;

$\%$ haemolysis $=($ ABS Sample - ABS $0 \%) /($ ABS100\% - ABS $0 \%)$

Where Abssample is the absorbance of supernatant of erythrocyte and nanoparticles suspension.

$\mathrm{Abs} 0 \%$ is the absorbance of supernatant of erythrocyte and PBS suspension.

Abs100\% is the absorbance of supernatant of erythrocyte and Triton X.

\section{In vitro cytotoxicity studies}

The monolayer cell culture was trypsinized and the cell count was adjusted to $1.0 \times 10^{5} \mathrm{cells} / \mathrm{ml}$ using DMEM medium containing $10 \%$ FBS. To each well of a 96 well microtitre plate, $100 \mu$ of the diluted cell suspension (approximately 10,000 cells/well) was added. After 24 hours, when a partial monolayer was formed, the supernatant was flicked off, the monolayer was washed once with medium and $100 \mu \mathrm{l}$ of different sample concentrations prepared in maintenance media were added per well to the partial monolayer in microtitre plates. The plates were then incubated at $37^{\circ} \mathrm{C}$ for $24 \mathrm{hrs}$ in $5 \% \mathrm{CO}_{2}$ atmosphere, and microscopic examination was carried out and observations recorded. After 24 hours, the sample solutions in the wells were discarded and 20 $\mu \mathrm{l}$ of MTT $(2 \mathrm{mg} / \mathrm{ml})$ in MEM-PR (MEM without phenol red)/PB was added to each well. The plates were gently shaken and incubated for 3 hours at $37^{\circ} \mathrm{C}$ in $5 \% \mathrm{CO}_{2}$ atmosphere. The supernatant was removed and $100 \mu \mathrm{l}$ of iso-propanol was added and the plates were gently shaken to solubilize the formed formazan. The absorbance was measured using a microplate reader at a wavelength of $540 \mathrm{~nm}$. The percentage growth inhibition was calculated using the following formula and concentration of drug or test samples needed to inhibit cell growth by $50 \%$ values were generated from the dose-response curves for each cell line.

$$
\% \text { Growth inhibition }=100-\frac{\text { Mean OD of individual test }}{\text { Mean OD of control group }} \text { X100 }
$$

\section{In vivo oral bioavailability and biodistribution studies}

Albino Wistar rats (Male and Female) weighing $200 \pm 20 \mathrm{~g}$ were used for oral bioavailability studies. All animal experiments were approved by Institutional Animal Ethical Committee, J.S.S College of Pharmacy, Ooty (Proposal no. JSSCP/IAEC/M.PHARM/ PH.CEUTICS/05/2015-16). All the rats were fasted for $12 \mathrm{~h}$ before the experiments but had free access to water. Groupings of animals have been done in the following manner. Group 1- Control group, Group 2Drug suspension treated group, Group 3- GMS Solid lipid nanoparticles treated group, Group 4- TP Solid lipid nanoparticles treated group. Drug suspension $(0.3 \% \mathrm{w} / \mathrm{v} \mathrm{CMC})$ and solid lipid nanoparticles were administered orally by oral feeding tube at dose of $1.6 \mathrm{mg}$ and $2.86 \mathrm{mg}$ of drug for F3 and F6 formulations respectively. Blood $(0.5 \mathrm{~mL})$ was collected by retro-orbital puncture at $0,0.30,1,4,8,12$ and 24 hours after administration separately. Blood samples were placed into eppendorf tubes containing $0.3 \mathrm{~mL}$ of anticoagulant (sodium citrate) solution and centrifuged immediately. After centrifugation, the plasma obtained was stored at $-20^{\circ} \mathrm{C}$ until further analysis. The organs like Heart, Brain, Kidneys, Liver, Lymph, Spleen, Ovaries and Uterus in female rats and Testis,Vas deferens in male rats were collected.The collected organs were washed with normal saline and homogenized and centrifuged for 10 mins at $10,000 \mathrm{rpm}$. Then the supernatant solution was analyzed by HPLC [11].

\section{Bioanalytical method development and analysis}

Reverse phase HPLC method is the most popular mode for analytical and preparative separations of the compounds in chemical, biological, pharmaceutical and food samples. In reversed phase mode, the stationary phase is non polar and the mobile phase is polar. The polar compounds gets eluted first in this mode and non polar compounds are retained for longer time. In present study, methods for the estimation of Simvastatin present in the blood plasma samples were developed and validated. For the estimation of Simvastatin in blood plasma, the chromatographic variables, namely $\mathrm{pH}$, solvent strength, solvent ratio, flow rate, addition of peak modifiers in mobile phase, nature of the stationary phase, detection wavelength and internal standard were studied and optimized for the separation and retention of the drug. The following are the optimized chromatographic conditions, preparation of standard and sample solutions and the methods used for the estimation of Simvastatin in plasma.

Chromatographic conditions: Shimadzu gradient HPLC system was used with following configurations: LC-20 AD 230V Solvent delivery system (Pump), Manual Injector $25 \mu \mathrm{l}$ (Rheodyne), SPD-M20A $230 \mathrm{~V}$ Photo diode array detector, Spinchrome data station, Stationary phase: Phenomenex Gemini C18 $(250 \times 4.6 \mathrm{~mm}$ i.d., $5 \mu)$, Mobile phase: Acetonitrile: $25 \mathrm{mM}$ Potassium Dihydrogen Orthophosphate 
Citation: Dasam U, Natarajan J, Karri VVSR, Wadhwani AD, Antony J, et al. (2016) Targeting Efficacy of Simvastatin for Hormone-Dependent Carcinomas through Solid Lipid Nanoparticles. J Nanomed Nanotechnol 7: 412. doi: 10.4172/2157-7439.1000412

(pH-3.5), Mobile phase ratio: 50:50, Flow rate: $0.5 \mathrm{ml} / \mathrm{min}$, Sample volume: $20 \mu \mathrm{l}$, Detection: $239 \mathrm{~nm}$, Data station: Spinchrome Solutions. The mobile phase was filtered through $0.22 \mu$ membrane and degassed using ultrasonicator. All the experiments were carried out at room temperature.

Preparation of Simvastatin standard stock solution: $10 \mathrm{mg}$ of Simvastatin was transferred into a $10 \mathrm{~mL}$ volumetric flask and the volume was made upto the mark with mobile phase to give $1 \mathrm{mg} / \mathrm{mL}$ $(1000 \mu \mathrm{g} / \mathrm{mL})$ solution. From this stock solution, $10 \mathrm{~mL}$ of $100 \mu \mathrm{g} / \mathrm{mL}$ solution was prepared and again from this solution $10 \mathrm{~mL}$ of $10 \mu \mathrm{g} / \mathrm{mL}$ was prepared.

Preparation of analytical calibration curve solutions: From the standard stock solution $0.25-2 \mu \mathrm{g} / \mathrm{ml}$ standard solutions were prepared and stored below $8^{\circ} \mathrm{C}$ until further analysis.

Preparation of blank plasma: Blank plasma $(0.2 \mathrm{~mL})$ was transferred into $2.0 \mathrm{ml}$ centrifuge tube and $0.2 \mathrm{~mL}$ of precipitating agent (10\% perchloric acid) was added. Finally made upto $2 \mathrm{~mL}$ with the mobile phase. The resulting solution was vortexed for 5 minutes and centrifuged at $4000 \mathrm{rpm}$ for 10 minutes. The supernatant layer was separated and analyzed.

Preparation of bioanalytical calibration curve samples: $0.2 \mathrm{~mL}$ of Rosvastatin solutions were transferred to $2.0 \mathrm{~mL}$ centrifuge tube respectively, to this $0.2 \mathrm{~mL}$ of plasma, $0.2 \mathrm{~mL}$ of precipitating agent were added. The resulting solution was vortexed for 5 minutes and centrifuged at $4000 \mathrm{rpm}$ for 10 minutes. The supernatant layer was separated and analyzed.

Preparation of plasma samples: Plasma samples $(0.2 \mathrm{~mL})$ obtained from study subjects was transferred into $2.0 \mathrm{~mL}$ centrifuge tube and $0.2 \mathrm{~mL}$ of precipitating agent was added. The resulting solution was vortexed for 5 minutes and centrifuged at $4000 \mathrm{rpm}$ for 10 minutes. The supernatant layer was separated and analysed.

Method of analysis: The bioanalytical calibration curve samples and plasma sample solutions were injected with above chromatographic conditions and the chromatograms were recorded. The quantification of the chromatogram was performed using peak area.

\section{Results and Discussion}

\section{Preformulation studies}

In DSC studies (Figure 1) it was concluded that the drug was entrapped into the polymer matrix without any chemical interaction. It was also further concluded that, the polymers were found to be compatible in entrapping the selected drug Simvastatin. The melting endotherm of the mixture proved that the crystalline form of the drug is converted to amorphous form and hence more amount drug solubilised in the lipid. As reported earlier, that rapid quenching of microemulsion does not allow the drug molecules dispersed in lipid phase to crystallize [12]. Furthermore, the presence of surfactants could not allow the drug to crystallize $[13,14]$.

The spectra obtained from FTIR studies at wavelength from $4000 \mathrm{~cm}^{-1}$ to $400 \mathrm{~cm}^{-1}$ are shown in Figure 2. After interpretation of the above spectra it was confirmed that there was no major shifting, loss or appearance of functional peaks between the spectra of drug, physical mixture of drug and lipid $\left(3548.09 \mathrm{~cm}^{-1}, 3451.46 \mathrm{~cm}^{-1}\right)$. From the spectra it was concluded that the drug was entrapped into the polymer matrix without any chemical interaction. From the IR study it was concluded that, the selected lipid tripalmitin was found to be compatible in entrapping the selected drug Simvastatin.

\section{Evaluation of solid lipid nanoparticles}

The significance of zeta potential is that its value can be related to the stability of colloidal dispersions. The zeta potential $(-5.16 \mathrm{mV})$ indicates the degree of repulsion between adjacent, similarly charged particles in dispersion and confirms the stability (Figure 3). The Polydispersity index (PI) is the measure of size distribution of the nanoparticle formulation. PI was measured using Malvern zetasizer. PI values range from 0.000 to 1.000 i.e. monodisperse to very broad particle size distribution. PI values of all the formulations indicate that particle size distribution was unimodel. The optimized TP-SLN (F6) batch having least particle size $177.4 \mathrm{~nm}$ with the PDI 0.650 .

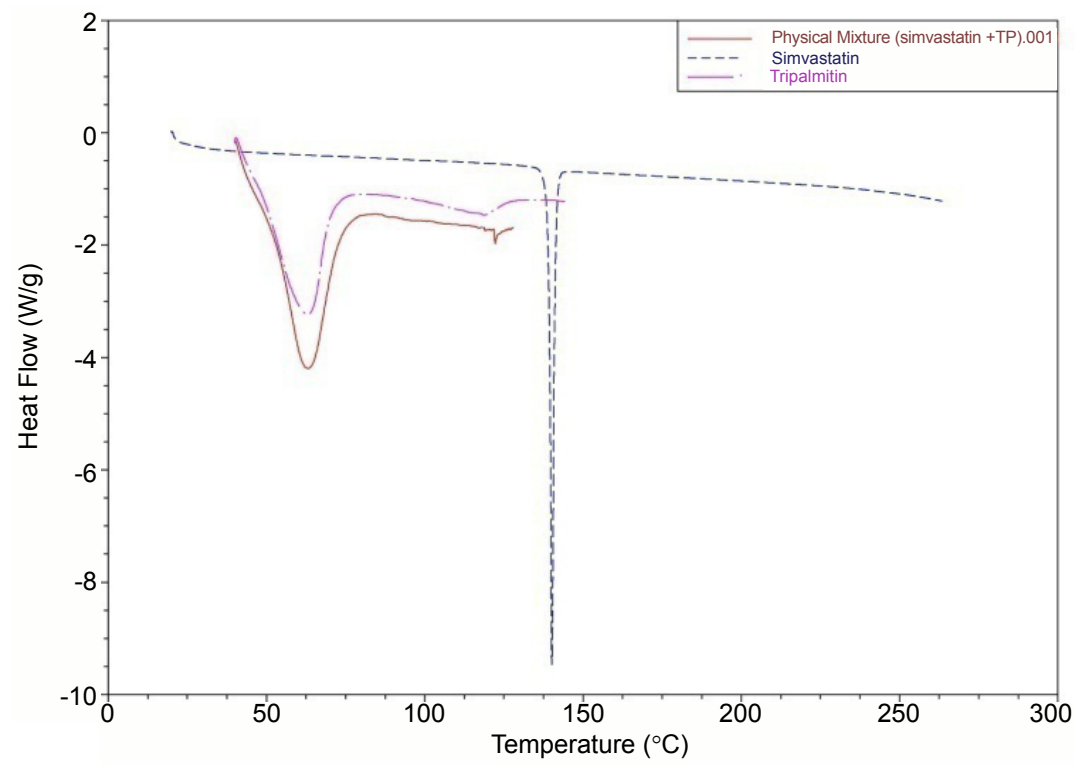

Figure 1: DSC thermograms of Tripalmitin, pure Simvastatin and TP-drug mixture. 
Citation: Dasam U, Natarajan J, Karri VVSR, Wadhwani AD, Antony J, et al. (2016) Targeting Efficacy of Simvastatin for Hormone-Dependent Carcinomas through Solid Lipid Nanoparticles. J Nanomed Nanotechnol 7: 412. doi: 10.4172/2157-7439.1000412

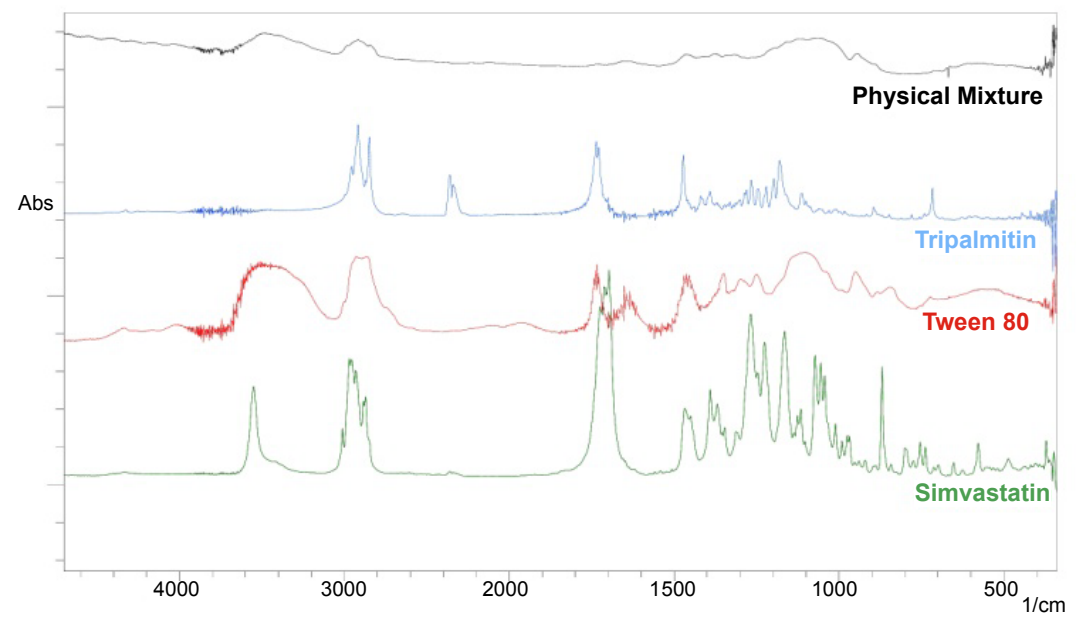

Figure 2: IR Spectra of Simvastatin, TP, Tween 80 and physical mixture.

Simvastatin is a hydrophobic drug with a log P value of 4.199 . The partition coefficient was found in order of TP(1) $>$ GMS (0.86) $>$ SA $(0.21)$. TP is more lipophilic of the 3 lipids and had higher affinity for Simvastatin.

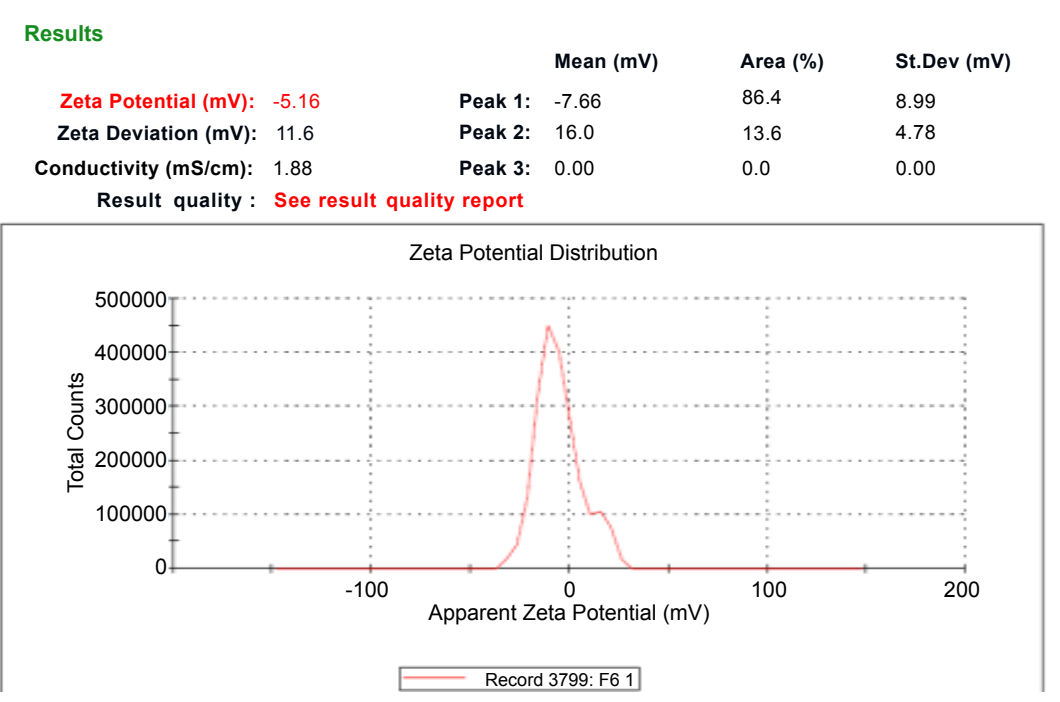

Figure 3: Zetapotential of F6 Formulation.

The lipid core was found to affect the extent of entrapment efficiency and drug loading. As observed with GMS-SLN and TP-SLN, the maximum entrapment efficiency was $78 \%$ (Batch F3) and $91.28 \%$ (Batch F6) respectively. The entrapment efficiency was higher when drug to lipid ratio was 1:10 (Batch F6) compared to drug to lipid ratio of 1:5 (Batch F3) i.e. when amount of drug taken for preparation of SLN was more $(10 \mathrm{mg})$, higher entrapment was obtained. TP-SLN showed higher entrapment efficiency compared to GMS-SLN because TP is more lipophilic than GMS and can accommodate more drugs in the lipid matrix. These results correlate well with partition coefficient studies. Similar results were obtained in case of drug loading, TP-SLN showed maximum drug loading of $15.15 \%$ (Batch F6) compared to GMS-SLN which showed maximum loading of $11.94 \%$. These results correlate well with partition coefficient studies

The External morphological studies (SEM) revealed that maximum nanoparticles were nearly spherical in shape (Figures 3 and 4). The nanoparticle size observed by SEM correlated well with the particle size measured by zeta sizer (Malvern instrument).
In vitro dissolution studies were carried out in Phosphate buffer pH 6.8 and results are shown in Figure 5. The release profiles indicate that SLN formulations showed a retarded release of the drug from the lipid matrix when compared with plain Simvastatin solution (SMSOL). The in vitro release data and graph of SLN formulations and SMSOL in Phosphate buffer $\mathrm{pH} 6.8$ is shown in Table 1 and Figure 5 . It was observed that SM-SOL showed $99.12 \%$ release in $1.5 \mathrm{~h}$ compared to $15.5 \%$ and $13.25 \%$ release for TP-SLN and GMS-SLN at the end of same time $(\mathrm{p}<0.05)$. This is due to fact that there is no barrier for diffusion at dialysis membrane interface for Simvastatin molecules. Hence, higher release was observed in case of SM-SOL. But, TP-SLN released the drug in a sustained manner.

In order to elucidate mode and mechanism of drug release, the invitro data was transformed and interpreted at graphical interface constructed using various kinetic models (Table 1). The in vitro release data obtained for TP-SLN formulation, in phosphate buffer $\mathrm{pH} 6.8$, was fitted into various kinetic models. The best linearity was obtained 
Citation: Dasam U, Natarajan J, Karri VVSR, Wadhwani AD, Antony J, et al. (2016) Targeting Efficacy of Simvastatin for Hormone-Dependent Carcinomas through Solid Lipid Nanoparticles. J Nanomed Nanotechnol 7: 412. doi: 10.4172/2157-7439.1000412

Page 6 of 7

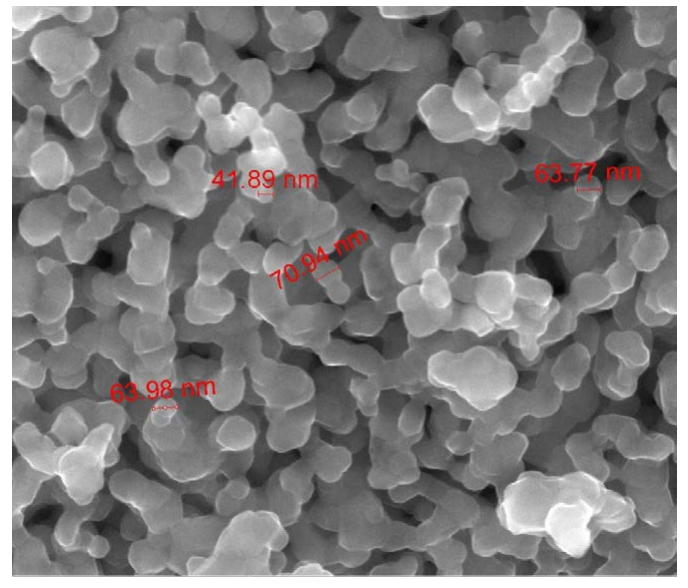

Figure 4: Scanning electron micrograph (SEM) of solid lipid nanoparticles.

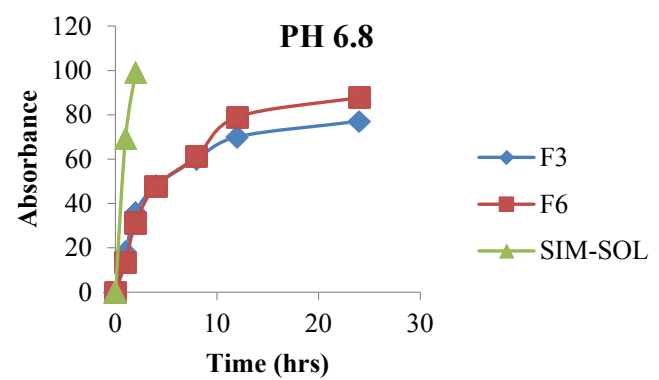

Figure 5: Comparative In vitro release profile in Phosphate buffer $\mathrm{pH} 6.8$.

\begin{tabular}{|c|c|c|c|c|}
\hline \multirow{2}{*}{ Formulation } & \multicolumn{4}{|c|}{$\mathbf{r}^{2}$} \\
\cline { 2 - 5 } & Zero order & First order & Higuchi's & Peppas \\
\hline TP-SLN & 0.6539 & 0.8191 & 0.8574 & 0.9313 \\
\hline
\end{tabular}

Table 1: Regression value for various kinetic models.

\begin{tabular}{|c|c|c|c|c|}
\hline \multirow{2}{*}{ Batch } & \multicolumn{4}{|c|}{$\%$ Haemolysis } \\
\cline { 2 - 5 } & $\mathbf{1 2 5} \boldsymbol{\mu \mathbf { g } / \mathbf { m l }}$ & $\mathbf{2 5 0} \boldsymbol{\mu g} / \mathbf{m l}$ & $\mathbf{5 0 0} \boldsymbol{\mu g} / \mathbf{m l}$ & $\mathbf{1 0 0 0} \boldsymbol{\mu g} / \mathbf{m l}$ \\
\hline F3 & $0.014 \pm 0.001$ & $0.021 \pm 0.001$ & $0.031 \pm 0.001$ & $0.042 \pm 0.001$ \\
\hline F6 & $0.011 \pm 0.001$ & $0.025 \pm 0.001$ & $0.035 \pm 0.001$ & $0.046 \pm 0.001$ \\
\hline
\end{tabular}

Table 2: \% Haemolysis of F3 and F6 formulations.

in Higuchi's plot for TP-SLN formulation indicating the release from matrix as a square root of time dependent process. The release exponent values 'n' for TP-SLN was found to be 0.619 . Since, the release exponent ' $n$ ' values were between $0.5-1$, it indicates that the SLN formulations undergo anomalous diffusion.

\section{Cytotoxicity studies}

Haemocompatability studies: Nanoparticles were subjected to rigorous blood biocompatibility tests. Erythrocyte-induced haemolysis in vitro can be considered to be a simple and reliable measure for estimating the membrane damage caused in vivo. Percent haemolysis was determined spectrophotometriclly, detecting plasma free haemoglobin derivatives after incubating the particles with blood and then separating the undamaged cells by centrifugation. Typically less than $5 \%$ haemolysis is considered acceptable for blood biocompatibility. The results of haemocompatability studies are shown in Table 2 . The concentration of $125-1000 \mu \mathrm{g} / \mathrm{ml}$ was subjected to determined percentage haemolysis. In this $1 \%$ triton X 100 was used as positive control and Saline water was as a negative control. Positive control showed percentage haemolysis of $3.714 \pm 0.65 \%$ where as negative control of $0.023 \pm 0.001 \%$. On increasing the concentration from 150 $\mu \mathrm{g} / \mathrm{ml}$ to $1000 \mu \mathrm{g} / \mathrm{ml}$, there was no significant increase in\% haemolysis.. The results indicated that the nanoparticles were haemocompatible and did not produce any toxic effects.

MTT assay: The percentage growth inhibition was calculated using the following formula and concentration of drug or test samples needed to inhibit cell growth by $50 \%$ values were generated from the dose-response curves for each cell line.

$$
\% \text { Growth inhibition }=100-\frac{\text { Mean OD of individual test group }}{\text { Mean OD of control group }} \times 100
$$

The IC 50 value of the formulation is about $0.165 \mathrm{mg} / \mathrm{ml}$ and IC 50 value of the drug is about $0.23 \mathrm{mg} / \mathrm{ml}$ (Figures 6 and 7).

\section{Bioanalytical method development and analysis}

Bioanalytical calibration curve of Simvastatin was prepared in mobile phase and peak area was calculated. The chromatograms of Simvastatin showed stable baseline. The regression equation in the range of $0.5-2 \mu \mathrm{g} / \mathrm{ml}$ was as follows: $\mathrm{y}=3730 \mathrm{x}+332.2, \mathrm{R}^{2}=0.990$. The concentration of Simvastatin was determined in plasma samples separated at different time intervals by HPLC analysis. The concentration of plasma samples was determined from the area of the chromatographic peak using the calibration graph.

The results of in vivo bioavailability and bio-distribution has been shown in Figures 8 and 9. The enhanced bioavailability by the SLNs formulation might be attributed to direct uptake of nanoparticles through the GI tract, increased permeability by surfactants, and decreased degradation and clearance. Firstly, the uptake of Simvastatin in the SLNs-encapsulated form could be up taken through the GI tract, where the particle size played a dominant role in absorption rate [15] The mechanisms of such uptake include diffusion of particles through mucus and accessibility to enterocyte surface, epithelial interaction and

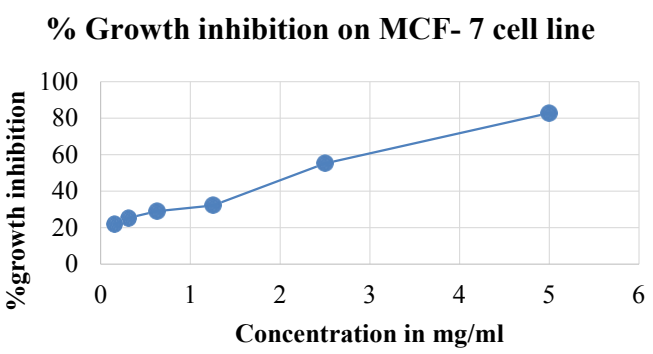

Figure 6: \%Growth inhibition of pure drug.

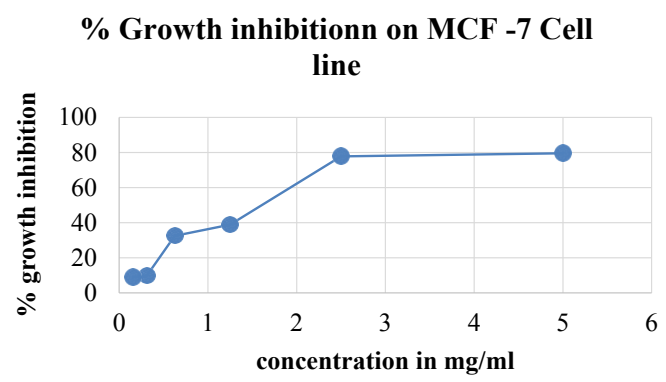

Figure 7: \%Growth inhibition of F6 formulation. 
Citation: Dasam U, Natarajan J, Karri VVSR, Wadhwani AD, Antony J, et al. (2016) Targeting Efficacy of Simvastatin for Hormone-Dependent Carcinomas through Solid Lipid Nanoparticles. J Nanomed Nanotechnol 7: 412. doi: 10.4172/2157-7439.1000412

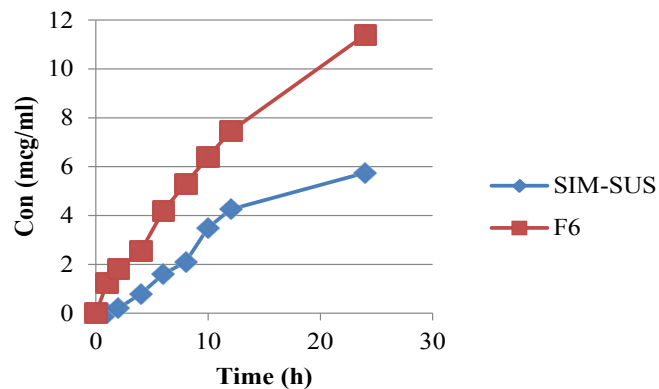

Figure 8: Concentration time profile after oral administration of Tripalmitin (TPSLN) and Simvastatin suspension (SM-SUSP).

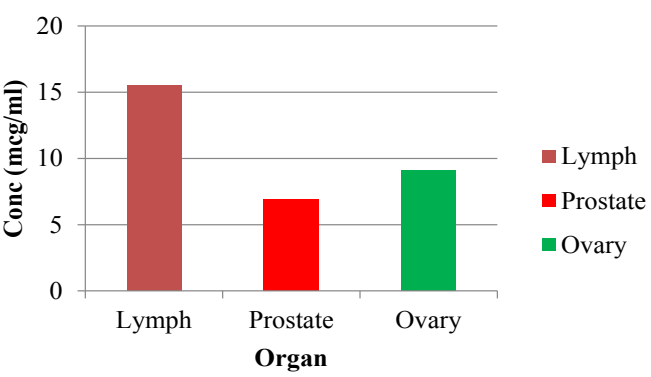

Figure 9: Simvastatin Distribution in various organs.

cellular trafficking, and exocytosis and systemic dissemination. The size of SLNs in the range of $20-500 \mathrm{~nm}$ allows the efficient uptake in intestine, particularly in the lymphoid sections of this tissue; therefore bypass the liver first-pass metabolism [16]. Secondly, the surfactants, such as Tween 80 , have contributed to an increase in the permeability of the intestinal membrane or improved the affinity between lipid particles and the intestinal membrane, and also may exhibit bioadhesion to the GI tract wall [17]. Thirdly, by incorporation into nanoparticles, Simvastatin can be embedded into a solid lipid matrix thus not only reducing its exposure to bacterium as well as enzymatic degradation during absorption process, but also offering a long time contact with the wall of intestine in vivo due to the nice adhesiveness of SLNs to the mucosal surface of intestine. Also, positively charged particles are better taken up by intestinal lymphatics than neutral or negatively charged particles $[16,17]$.

Apical potential of epithelial cells of gastrointestinal tract as well as other cells in the body possess a negative charge on their surface due to the presence of negatively charged proteins on the outer membrane of the cells there by better permeability and uptake would occur for positively charged colloidal particles due to electrostatic attraction between oppositely charged surfaces $[17,18]$. In addition, Simvastatin-SLNs could provide Simvastatin with a long circulation effect in vivo with sustained-release property, which prolonged the drug residence time in systematic circulation and resulted in better bioavailability $[19,20]$.

In biodistribution study it is clear that SIM-SLNs showed a comparitive better amount of drug sistrubtuion to organs like lymph, prostate and ovary. The drug was bound to the tissues of various organs and the availability of the drug shows that the formulation is targeted to the specific sites.

\section{Conclusion}

In this study, we have described the preparation and characterization of Folate conjugated Simvastatin SLNs. In MCF7 Human Breast carcinoma cells, FCSIM-SLNs showed greater cytotoxicity than free drug solutions. The SLNs showed a significant increase in oral bioavailability compared to pure drug suspension. Higher relative bioavailability would be due to avoidance of firstpass hepatic metabolism by intestinal lymphatic transport, which circumvents the liver. SLNs provided sustained release of the drugs, and these systems are the preferred drug carriers for lipophilic drugs to overcome the oral bioavailability problem of drug and to increase targeting efficacy to the specific organs as breast, prostate and ovary.

\section{References}

1. Herington AC, Chopin LK, Jeffery $P$, de Amorim L, Veveris-Lowe T, et al. (2010) Hormone-dependent cancers: new approaches to identification of potential diagnostic and/or therapeutic biomarkers. Asia Pac J Mol Biol Bio 18: 63-66.

2. McGuire A, Brown JA, Malone C, McLaughlin R, Kerin MJ (2015) Effects of age on the detection and management of breast cancer. Cancers 22: 908-929.

3. Ruddon RW (2007) Cancer biology. Oxford University Press, USA.

4. Illingworth DR (1987) Lipid-lowering drugs. Drugs 33: 259-279.

5. Vinayak S, Kurian AW (2009) Statins may reduce breast cancer risk, particularly hormone receptor-negative disease. Curr Breast Cancer Rep 1: 148-156.

6. Liang XJ, Chen C, Zhao Y, Wang PC (2010) Circumventing tumor resistance to chemotherapy by nanotechnology. Methods Mol Biol 596: 467-488.

7. Wu TT, Zhou SH (2015) Nanoparticle-based targeted therapeutics in headand-neck cancer. Int J Med Sci 12: 187.

8. Narvekar M, Xue HY, Eoh JY, Wong HL (2014) Nanocarrier for poorly watersoluble anticancer drugs-barriers of translation and solutions. AAPS Pharm Sci Tech 15: 822-833.

9. Sood S, Jawahar N, Jain K, Gowthamarajan K, NainarMeyyanathan S (2013) Olanzapine loaded cationic solid lipid nanoparticles for improved ora bioavailability. Curr Nano 9: 26-34.

10. Müller RH, Maaßen S, Schwarz C, Mehnert W (1997) Solid lipid nanoparticles (SLN) as potential carrier for human use: interaction with human granulocytes. J Control Rel 47: 261-269.

11. Padhye SG, Mangal S (2013) Simvastatin Solid Lipid Nanoparticles for Ora Delivery: Formulation Development and In vivo Evaluation. Indian J Pharm Sci 75: 591-599.

12. Cavalli R, Aquilano D, Carlotti ME, Gasco MR (1995) Study by X-ray powder diffraction and differential scanning calorimetry of two model drugs phenothiazine and nifedipine, incorporated into lipid nanoparticles. Eur J Pharm Biopharm 41: 329-333.

13. zur Mühlen A, Schwarz C, Mehenert W (1998) Solid lipid nanoparticles (SLN) for controlled drug delivery-drug release and release mechanisms. Eur J Pharm Biopharm 45: 149-155.

14. Freitas C, Müller RH (1999) Correlation between long-term stability of solid lipid nanoparticles (SLNTM) and crystallinity of the lipid phase. Eur J Pharm Biopharm 47: 125-132.

15. Hussain N, Jaitley V, Florence AT (2012) Recent advances in the understanding of uptake of microparticulates across the gastrointestinal lymphatics. Adv Drug Deliv Rev 50: 107-142.

16. Huang G, Zhang N, Bi X, Dou M (2008) Solid lipid nanoparticles of temozolomide: Potential reduction of cardiac and nephric toxicity. Int J Pharm 355: 314-320.

17. Manjunath K, Venkateswarlu V (2005) Pharmacokinetics, tissue distribution and bioavailability of clozapine solid lipid nanoparticles after intravenous and intraduodenal administration. J Control Rel 107: 215-228.

18. Porter CJH, Pouton CW, Cuine JF, Charman WN (2008) Enhancing intestinal drug solubilisation using lipid-based delivery systems. Adv Drug Deliv Rev 60: 673-691.

19. Olbrich C, Müller RH (1999) Enzymatic degradation of SLN-effect of surfactant and surfactant mixtures. Int J Pharm 180: 31-39.

20. Olbrich C, Kayser O, Müller RH (2002) Lipase degradation of Dynasan 114 and 116 solid lipid nanoparticles (SLN)-effect of surfactants, storage time and crystallinity. Int J Pharm 237: 119-128. 